\title{
Colored noise driven systems with inertia
}

\author{
L.H'walisz $^{1}$, P. Jung ${ }^{1}$, P. Hänggi ${ }^{1}$, P. Talkner ${ }^{2}$, and L. Schimansky-Geier ${ }^{3}$ \\ ${ }^{1}$ Institut für Mathematik, Universität Augsburg, Federal Republic of Germany \\ ${ }^{2}$ Institut für Theoretische Physik, Universität Basel, Switzerland \\ ${ }^{3}$ Sektion Physik, Humboldt-Universität zu Berlin, German Democratic Republic
}

Received May 3, 1989; revised version July 13, 1989

\begin{abstract}
We present a novel approximation scheme, termed unified colored noise approximation (UCNA), for colored Gaussian noise driven nonlinear systems with inertia. This approximation allows one to evaluate static (stationary distributions, moments) as well as dynamical quantities (correlation functions) for small-to-moderate-to-large values of the correlation time. The approximation replaces a three-dimensional Markovian process by a reduced, two-dimensional Markovian dynamics with new drift and diffusion coefficients. For a harmonic potential the stationary moments are reproduced exactly. Most importantly, we present a criterion involving the noise strength $D$, the friction strength $\gamma$ and the noise color $\tau$, which describes the region of validity of UCNA in the parameter space given by $(D, \tau, \gamma)$. At small $\tau$-values we contrast the UCNA with the well-known small $\tau$ approximation. In order to have a comparison on analytical grounds, we test the static and dynamical predictions of UCNA versus the well-known analytical results obtained from a three-dimensional Ornstein-Uhlenbeck process.
\end{abstract}

\section{Introduction}

The study of noise driven nonlinear dynamical systems is attracting rapidly growing interest. Especially in the theory of non-equilibrium systems, where the macrovariables obey some nonlinear equations of motion [1], noise plays an important role. Only in the presence of noise the system can surmount potential barriers and is thus able to explore the whole potential landscape, thereby attaining different macrostates [2]. For the realistic modeling of physical systems, however, it is necessary to take into account finite (but not necessarily small) correlation times of the noise. Such a noise with a frequency dependent spectral density is termed "colored noise".

Recently the importance of colored noise in nonequilibrium quantum optical systems like dye lasers [3], laser gyros [4] and bistable optical devices [5] was emphasized in a large number of papers. The majority of these papers assumes a situation where one relevant variable obeying an overdamped equation of motion is coupled to a colored noise force. Because of the non-Markovian (and non-equilibrium) properties even this situation is difficult to describe in analyt- ical form. Approximation schemes have thus been developed for the limiting cases of vanishing small [6] and extremely large [7] correlation times of the noise. For the relevant regime of correlation times of the order of the typical time scale of the system variables the "decoupling-theory" [8] as well as the recently developed "unified colored noise approximation (UCNA)" [3 m, 9] are applicable besides numerical methods $[10,11]$.

In a number of systems (e.g. the resistively shunted Josephson-junction [12]), however, the simplifying assumption of an overdamped dynamics of the relevant variable is not valid, i.e. the effects of finite inertia must be accounted for. For the particular case of a bistable potential a system has been studied recently by Moss et al. [13] using analog simulation techniques. Local approximative solutions for this case were reported in $[14,15]$ in the case of small $\tau$. Fronzoni et al. [16] have worked out an extended version of the decoupling theory [8] for inertial systems and demonstrated good agreement between the analog simulation experiments and the theory. Very recently Marchesoni et al. [17] have considered the weak damping limit of a bistable system driven by colored 
noise. In contrast to [13] and [16], they focus on dynamical quantities as e.g. the spectral densities, correlation functions and escape times. In the present paper we discuss an approximation scheme for nonlinear systems with inertia which are driven by an external Gaussian colored noise. This objective presents an extension of the overdamped UCNA discussed previously [9]. The novel approximation scheme reduces a three-dimensional Markovian process to an approximate two-dimensional Markovian process. For its regime of validity we give an explicit condition in the three-dimensional parameter space consisting of the noise strength $D$, the noise correlation time $\tau$ and the damping $\gamma$. In contrast to the small correlation time expansions [6], the validity of our novel scheme is not restricted to small values of $\tau$, but also allows a study of small, moderate and large $\tau$ values. Furthermore, being a Markovian approach to a nonMarkovian process, our approximation scheme enables one to calculate dynamical quantities such as correlation functions and relaxation times.

Because exact analytical solutions for non-linear three-dimensional Markovian processes which lack detailed balance symmetry are not available, we test our novel approximation scheme for the exactly solvable case of a parabolic potential. Our paper is organized as follows: In the next section we introduce the system and discuss its physical relevance in the context of equilibrium and non-equilibrium systems. In Sect. 3 we present our novel approximation scheme for a nonlinear three-dimensional system leading to an approximate two-dimensional Markovian process. The exact results for the parabolic potential with inertia are discussed in Sect. 4. The UCNA is then tested in Sect. 5 against the exact analytical results for the three-dimensional Ornstein-Uhlenbeck dynamics by comparing moments, correlation functions and eigenvalues of both processes. Moreover, the high friction limit is discussed in Sect. 5.4.

\section{Colored noise with inertia}

The Brownian motion in a potential $V(x)$ is properly described in terms of Langevin equations, which are Newton's equations of motion supplemented by a stochastic force. Assuming a correlated stochastic force obeying the fluctuation-dissipation-theorem we are forced to include memory-damping for equilibrium systems, where the noise stems from the same heat bath into which excess energy from the system dissipates, i.e.

$m \ddot{x}(t)+\int_{0}^{t} \beta(t-s) \dot{x}(s) \mathrm{d} s+V^{\prime}(x(t))=m \Gamma(t)$, where the Gaussian noise $\Gamma(t)$ of vanishing mean obeys the fluctuation-dissipation-theorem

$\langle\Gamma(t) \Gamma(s)\rangle=\frac{k T}{m} \beta(t-s)$.

Hereby we assume the notation $m$ for the mass, $T$ for the temperature, $V(x)$ is the potential and $k$ denotes the Boltzmann constant.

In non-equilibrium systems noise and dissipation may have a different origin such that no fluctuationdissipation relation holds. Thus, following the concept of an external noise force driving the motion in the potential $V(x)$ the Langevin-equation reads $[13,16]$

$m \ddot{x}(t)+\beta \dot{x}(t)+V^{\prime}(x(t))=m \Gamma(t)$

$\left\langle\Gamma(t) \Gamma\left(t^{\prime}\right)\right\rangle=\frac{\theta}{m} \exp \left\{-\frac{1}{\tau}\left|t-t^{\prime}\right|\right\}$

$\langle\Gamma(t)\rangle=0$.

Here we have already specified the correlation function to be of exponential form with the correlation time denoted by $\tau$ and the noise strength by $D \equiv \theta / m$.

In the present paper we discuss the nonequilibrium system (2.2). In contrast to equilibrium systems (2.1 a), possessing the well known canonical equilibrium distribution

$P_{\mathrm{eq}}(x, \dot{x}=v)=Z^{-1} \exp \left[-\frac{V(x)}{k T}-\frac{m v^{2}}{2 k T}\right]$,

the stationary distribution of (2.2) is generally not known analytically. In the overdamped case $(\gamma \rightarrow \infty)$ it has been demonstrated, that the stationary distribution can change its shape qualitatively (new peaks [3 f], shifted peaks [ $3 \mathrm{~m}, 10 \mathrm{a}, \mathrm{d}]$ formation of craters $[7 \mathrm{c}, 18]$ ) upon varying the correlation time $\tau$, thereby changing drastically $[7 \mathrm{c}]$ also the dynamical properties such as e.g. the escape times (via the escape path). All these phenomena are also expected to occur in systems with inertia, such as (2.2).

The non-Markovian stochastic process (2.2) can be embedded into a three-dimensional Markovian dynamics, which after a normalization, i.e. $D=\frac{\theta}{m}, \gamma=\frac{\beta}{m}$, $U(x)=\frac{V(x)}{m}$ and with the force-field $f(x)=U^{\prime}(x)$ reads

$\dot{x}=v$

$\dot{v}=-\gamma v+f(x)+\varepsilon$

$\dot{\varepsilon}=-\frac{1}{\tau} \varepsilon+\frac{1}{\tau} \sqrt{D} \xi(t)$, 
with the Gaussian white noise $\xi(t)$ obeying

$\left\langle\xi(t) \xi\left(t^{\prime}\right)\right\rangle=2 \delta\left(t-t^{\prime}\right)$

$\langle\xi(t)\rangle=0$.

The corresponding Fokker-Planck equation (FPE) for $P(x, v, \varepsilon, t)$, i.e.

$$
\begin{aligned}
\frac{\partial P}{\partial t}= & -\frac{\partial}{\partial x} v P+\gamma \frac{\partial}{\partial v} v P-f(x) \frac{\partial}{\partial v} P-\varepsilon \frac{\partial}{\partial v} P \\
& +\frac{1}{\tau} \frac{\partial}{\partial \varepsilon} \varepsilon P+\frac{D}{\tau^{2}} \frac{\partial^{2}}{\partial \varepsilon^{2}} P,
\end{aligned}
$$

does not obey detailed balance in general. However, it may be solved numerically using for example finite difference methods (a point of future investigations). The approximation scheme presented in the next section provides an approximate two-variable FokkerPlanck equation, which in principle may be solved numerically with the matrix continued fraction (MCF) technique developed by Risken and Vollmer [11].

For a parabolic potential $U(x)=1 / 2 \omega_{0}^{2} x^{2}$ the stationary probability distribution $P_{\mathrm{st}}(x, v, \varepsilon)$ of $(2.5)$ can be calculated explicitly, yielding

$P_{\mathrm{st}}(x, v, \varepsilon)=Z^{-1} \exp \left(-\frac{\Phi(x, v, \varepsilon)}{D}\right)$

where $Z$ is a normalisation constant and $\Phi(x, v, \varepsilon)$ is given by

$$
\begin{aligned}
\Phi(x, v, \varepsilon)= & \frac{1}{2} \gamma \omega_{0}^{2}\left(1+\tau^{2} \omega_{0}^{2}\right) x^{2}+\gamma^{2} \tau^{2} \omega_{0}^{2} x v \\
& +\frac{1}{2} \gamma\left((1+\gamma \tau)^{2}+\tau^{2} \omega_{0}^{2}\right) v^{2} \\
& +\frac{1}{2} \tau(1+\gamma \tau) \varepsilon^{2}-\gamma \tau(1+\gamma \tau) \varepsilon v-\gamma \tau^{2} \omega_{0}^{2} x \varepsilon .
\end{aligned}
$$

Introducting a new variable $q$, defined by

$q \equiv-\gamma v+\varepsilon-\frac{\gamma \tau \omega_{0}^{2} x}{1+\gamma \tau}$

(2.6b) can be recast into a cross-correlation-free form, i.e.

$$
\begin{aligned}
\Phi(x, v, q)= & \frac{1}{2} \tau(1+\gamma \tau) q^{2}+\frac{1}{2}\left(1+\gamma \tau+\tau^{2} \omega_{0}^{2}\right) \gamma v^{2} \\
& +\frac{1}{2} \gamma \omega_{0}^{2}\left(1+\frac{\tau^{2} \omega_{0}^{2}}{1+\gamma \tau}\right) x^{2} .
\end{aligned}
$$

\section{The approximation scheme}

The approximation scheme presented in this section is closely related to that proposed in [9] for the overdamped case $(\gamma \rightarrow \infty)$. For a nonlinear force-field $f(x)$, we introduce the new variable

$q \equiv-\gamma v+\varepsilon+\frac{\gamma \tau f(x)}{1+\gamma \tau}$,

which coincides with (2.7) in the linear case. This transformation (3.1) follows from $[19,20]$, wherein factorizations of the steady state probability are investigated. In particular, for a parabolic potential the stationary probability factorizes exactly in the variables $x, q$ and $v$. An adiabatic elimination scheme for $q$ yields already the exact stationary probability in $x$ and $v$ (see below). In the case of a nonlinear force-field the factorization is not exact, and an adiabatic elimination does no longer provide the exact probability. The error of the adiabatic approach, however, is only due to the non-linearity of the force-field. Thus for our adiabatic approximation scheme the set of variables $\{x, v, q\}$ is more suitable than $\{x, v, \varepsilon\}$.

Using (3.1), (2.4 a) can be recast into the following form

$\dot{x}=v$

$\dot{v}=q+\frac{f(x)}{1+\gamma \tau}$

$\dot{q}=-\left(\gamma+\frac{1}{\tau}\right) q+\frac{\gamma}{\tau}\left[\frac{\tau^{2}}{1+\gamma \tau} f^{\prime}(x)-1\right] v+\frac{\sqrt{D}}{\tau} \xi(t)$.

With a time scale transformation, i.e. $\bar{t}=t / \sqrt{\tau},(3.2 \mathrm{c})$ reads

$$
\begin{aligned}
\dot{q}= & -\left(\gamma \sqrt{\tau}+\frac{1}{\sqrt{\tau}}\right) q+\frac{\gamma}{\tau^{1 / 2}}\left[\frac{\tau^{2}}{1+\gamma \tau} f^{\prime}(x)-1\right] v \\
& +\frac{\sqrt{D}}{\tau^{3 / 4}} \bar{\xi}(\bar{t})
\end{aligned}
$$

where

$\left\langle\bar{\xi}(\bar{t}) \bar{\zeta}\left(\bar{t}^{\prime}\right)\right\rangle=2 \delta\left(\bar{t}-\bar{t}^{\prime}\right)$.

The dots in $(3.3 \mathrm{a}, \mathrm{b})$ indicate the derivative with respect to $\bar{t}$. In contrast to [9] the damping coefficient $\Gamma \equiv \tau^{-1 / 2}+\gamma \tau^{1 / 2}$ in (3.3a) is independent of the state variables $x$ and $v$. Note that $\Gamma$ becomes large in the limiting cases of both small and large correlation times $\tau$ of the noise. Thus $\dot{q}$ can be neglected in both limiting cases (i.e. $q$ is eliminated adiabatically, both for $\tau \rightarrow 0$ 
and $\tau \rightarrow \infty)$. After rescaling to the original time scale $t$ (3.3a) becomes (with $\dot{q}$ set equal to zero)

$q=\frac{\gamma}{1+\gamma \tau}\left[\frac{\tau^{2}}{1+\gamma \tau} f^{\prime}(x)-1\right] v+\frac{\sqrt{D}}{1+\gamma \tau} \xi(t)$.

Using (3.4), (3.2a, b) can be written as

$\dot{x}=v$

$\dot{v}=-\frac{\gamma}{1+\gamma \tau}\left[1-\frac{\tau^{2}}{1+\gamma \tau} f^{\prime}(x)\right] v+\frac{f(x)}{1+\gamma \tau}+\frac{\sqrt{D}}{1+\gamma \tau} \xi(t)$

being valid on times $\bar{t}>\Gamma^{-1}$, i.e. for small and large $\tau$, or more precisely

$t>\frac{\tau}{1+\gamma \tau}$,

and in regions of parameter space $(D, \tau, \gamma)$ obeying (see Appendix C)

$\frac{(D \tau)^{1 / 2} \gamma}{(1+\gamma \tau)^{3 / 2}} \ll 1$

A contour plot in the $\gamma-\tau$ place for different values of (3.6b) is shown in Fig. 1.

The corresponding two-variable FPE

$$
\begin{aligned}
\frac{\partial}{\partial t} P= & -\frac{\partial}{\partial x} v P+\frac{\gamma}{1+\gamma \tau}\left[1-\frac{\tau^{2}}{1+\gamma \tau} f^{\prime}(x)\right] \frac{\partial}{\partial v} v P \\
& -\frac{f(x)}{1+\gamma \tau} \frac{\partial}{\partial v} P+\frac{D}{(1+\gamma \tau)^{2}} \frac{\partial^{2}}{d v^{2}} P
\end{aligned}
$$

may be solved for a general potential $f(x)$ in terms of matrix continued fractions [11].

Our main results $(3.5 \mathrm{a}, \mathrm{b}, 3.7)$ approximate a twodimensional non-Markovian process (2.2) by a two-dimensional Markovian process. Thus, this approximation does not only allow the calculation of stationary distributions and moments, but in addition describes correctly (within its regime of validity) the dynamical properties of the non-Markovian process (2.2).

In the overdamped limit $(\gamma \rightarrow \infty) \dot{v}$ can also be neglected (i.e. is eliminated adiabatically) in (3.5). This is seen best after performing a time scale transformation $\hat{t}=t / \sqrt{1+\gamma \tau}$ in (3.5). In the original time scale we obtain the result of [9], i.e.

$\dot{x}=\frac{f(x)}{\gamma-\tau f^{\prime}(x)}+\frac{\sqrt{D}}{\gamma-\tau f^{\prime}(x)} \xi(t)$,

which is valid in regions of $x$-space satisfying [9]

$D^{1 / 2}\left|f^{\prime}(x) / f(x)\right| \ll \gamma \tau^{-1 / 2}-\tau^{1 / 2} f^{\prime}(x)$.
Our novel approximation scheme, covering the results of [9] in the overdamped limit $(\gamma \rightarrow \infty)$, presents thereby an extension of the "Unified Colored Noise Approximation" (UCNA) put forward in [9].

\section{Exactly solvable test model}

For the parabolic potential

$$
U(x)=\frac{1}{2} \omega_{0}^{2} x^{2}
$$

the stochastic process $(2.4 \mathrm{a}, \mathrm{b})$ becomes a three-dimensional $(N=3)$ Ornstein-Uhlenbeck process (OUprocess) which is exactly solvable [21]. In the following we consider moments, correlation functions, eigenfunctions and the eigenvalues of the FPE (2.5). For the eigenvalues we solve in Appendix A the more general case of a $\mathrm{N}$-dimensional OU-process and apply the results to our special three-dimensional case.

\subsection{Moments and stationary correlation functions}

The stationary moments of the distribution (2.6a) may be calculated without solving the full FPE (2.5) for the stationary distribution by using the identities

$$
\left\langle\underline{L}_{\mathrm{FP}}^{\dagger} x^{n} v^{m} \varepsilon^{l}\right\rangle_{\mathrm{st}}=0, \quad n, m, l=0,1,2 \ldots
$$

The index "st" at the right bracket indicates an average over the stationary distribution of (2.5). $\underline{L}_{\mathrm{FP}}^{\dagger}$ denotes the adjoint Fokker-Planck-operator.

For the moments $\left\langle x^{2}\right\rangle_{\mathrm{st}},\left\langle v^{2}\right\rangle_{\mathrm{st}},\langle x \varepsilon\rangle_{\mathrm{st}}$ and $\langle x v\rangle_{\mathrm{st}}$ we find the exact results (see also (2.8)),

$$
\begin{aligned}
& \left\langle x^{2}\right\rangle_{\mathrm{st}}=\frac{D}{\gamma \omega_{0}^{2}}\left(1-\tau^{2} \frac{\omega_{0}^{2}}{1+\gamma \tau+\omega_{0}^{2} \tau^{2}}\right) \\
& \left\langle v^{2}\right\rangle_{\mathrm{st}}=\frac{D / \gamma}{1+\gamma \tau+\omega_{0}^{2} \tau^{2}} \\
& \langle x \varepsilon\rangle_{\mathrm{st}}=\tau \frac{D}{1+\gamma \tau+\omega_{0}^{2} \tau^{2}} \\
& \langle x v\rangle_{\mathrm{st}}=0 .
\end{aligned}
$$

The moments $(4.3 \mathrm{a}, \mathrm{b})$ and $(4.5)$ are also recovered from the approximative process (3.5) (see Sect. 5). Equation (4.4) is needed for the calculation of the correlation function $\Phi_{x x}(t)$.

The temporal decay of fluctuations is described by correlation functions. The normalized stationary position-position correlation function defined by

$\phi_{x x}(t)=\frac{\langle x(t) x(0)\rangle_{\mathrm{st}}}{\left\langle x^{2}\right\rangle_{\mathrm{st}}}, \quad \phi_{x x}(0)=1$, 
is given by [21]

$$
\begin{aligned}
& \phi_{x x}(t)=\left(\frac{\gamma}{2 \omega} \sin (\omega t)+\cos (\omega t)\right) \\
& \cdot \exp \left(-\frac{\gamma}{2} t\right)+\frac{\langle\varepsilon x\rangle_{\mathrm{st}}}{\left\langle x^{2}\right\rangle_{\mathrm{st}}\left(\alpha^{2}+\omega^{2}\right)} \\
& \cdot\left[\exp \left(-\frac{1}{\tau} t\right)-\exp \left(-\frac{\gamma}{2} t\right)\left(\frac{\alpha}{\omega} \sin (\omega t)+\cos (\omega t)\right)\right] \\
& \text { for } \gamma<2 \omega_{0} .
\end{aligned}
$$

In the overdamped case $\left(\gamma>2 \omega_{0}\right) \omega$ has to be substituted by $i \omega$.

In the aperiodic limit case $\left(\gamma=2 \omega_{0}\right)$ we find:

$$
\begin{aligned}
& \phi_{x x}(t)=\left(\frac{\gamma}{2} t+1\right) \exp \left(-\frac{\gamma}{2} t\right)+\frac{\langle\varepsilon x\rangle_{\mathrm{st}}}{\left\langle x^{2}\right\rangle_{\mathrm{st}} \alpha^{2}} \\
& \cdot\left[\exp \left(-\frac{1}{\tau} t\right)-\exp \left(-\frac{\gamma}{2} t\right)(\alpha t+1)\right]
\end{aligned}
$$

where

$\alpha=\frac{\gamma}{2}-\frac{1}{\tau} \quad$ and $\quad \omega=\sqrt{\left|\omega_{0}^{2}-\frac{1}{4} \gamma^{2}\right|}$.

\subsection{Eigenvalues}

The eigenvalues and eigenfunctions of a $\mathrm{N}$-dimensional OU-process cannot be found in reviews or standard text-books $[2,11 \mathrm{c}, 28]$ and are thus derived explicitly in Appendix A. Specifying the results for the threedimensional FPE (2.5) with the parabolic potential (4.1) we obtain

$\lambda_{l ; m ; n}=-\left(l \Lambda_{1}+m \Lambda_{2}+n A_{3}\right), \quad l, m, n=0,1,2 \ldots$

where

$\Lambda_{1}=-\frac{1}{\tau}$

and

$$
A_{2 ; 3}=-\frac{\gamma}{2} \pm \sqrt{\frac{\gamma^{2}}{4}-\omega_{0}^{2}}
$$

Though it is also possible to determine the corresponding eigenfunctions, we restrict ourselves to the eigenvalues.

\section{UCNA versus exact results of the test model}

In this section we compare stationary probability densities, eigenvalues and correlation functions of the OU-process discussed in Sects. 2 and 4 versus the corresponding approximative, but analytic results obtained by using the approximation scheme in Sect. 3 . The UCNA-Fokker-Planck equation (3.7) with the parabolic potential (4.1) corresponds to a two-dimensional OU-process and is thus exactly solvable.

\subsection{Eigenvalues}

The dynamical characteristics of a stochastic process described by an autonomous FPE (i.e. no external time dependend coherent excitation present) may be expressed by the eigenvalues and eigenfunctions of the Fokker-Planck operator and its adjoint operator. The agreement between the eigenvalues presents therefore an important benchmark for the quality of a lower dimensional Fokker-Planck approach like the UCNA. The eigenvalues of the UCNA-FPE with the parabolic potential (4.1) may be determined using the technique presented in Appendix A. We find

$$
\lambda_{m ; n}^{\mathrm{UCNA}}(\tau)=-\left(m \Sigma_{2}(\tau)+n \Sigma_{3}(\tau)\right)
$$

where

$$
\begin{aligned}
& \Sigma_{2 ; 3}(\tau)= \\
& -\frac{\gamma}{2 \Omega^{2}}\left(\Omega+\tau^{2} \omega_{0}^{2} \pm \sqrt{\left(\Omega+\tau^{2} \omega_{0}^{2}\right)^{2}-4 \Omega^{3} \frac{\omega_{0}^{2}}{\gamma^{2}}}\right),
\end{aligned}
$$

and $\Omega$ is defined as

$\Omega=1+\gamma \tau$.

Note that with $(4.8 \mathrm{c})$

$\Lambda_{2 ; 3}=\Sigma_{2 ; 3}(\tau=0)$.

For small correlation times $\tau^{2} \omega_{0}^{2} \ll 1$ and moderate damping $\gamma \ll 1 / \tau$ (for instance $\tau=0.1$ and $\gamma=1$ in normalized units [23]), we recover a part of the spectrum of the exact solution (see (4.8)), i.e.

$\lambda_{m ; n}^{\mathrm{UCNA}} \rightarrow \lambda_{l=0 ; m ; n}$ for $\tau^{2} \omega_{0}^{2} \ll 1$ and $\gamma \ll 1 / \tau$.

The eigenvalues $\lambda_{l ; m=0 ; n=0}=l / \tau(4.8 \mathrm{a})$ are large in the limit $\tau \rightarrow 0$ and thus influence only the short-time behavior of dynamical observables. On the short-time 
scale $t<\tau /(1+\gamma \tau)$, see (3.6a), our adiabatic approach is of course not valid. The behavior for moderate-tolarge times is governed by the relevant eigenvalues $\lambda_{l=0 ; m ; n}$ having a smaller real part.

In the case $\tau \gamma \gg 1$, e.g. small $\tau$ and large $\gamma$ or large $\tau$ and moderate-to-large $\gamma$ (for instance $\tau=0.1, \gamma=100$ or $\tau=100, \gamma=1$ ), we obtain for the approximated eigenvalues

$\lambda_{l ; n}^{\mathrm{UCNA}} \cong l \frac{1}{\tau}+n \frac{\omega_{0}^{2}}{\gamma} \quad$ for $\tau \gamma \gg 1$

The exact eigenvalues for high friction read

$\lambda_{l ; m ; n} \cong l \frac{1}{\tau}+n \frac{\omega_{0}^{2}}{\gamma}+m\left(\gamma-\frac{\omega_{0}^{2}}{\gamma}\right), \quad$ as $\gamma \gg 1$.

Again we find that the largest eigenvalues $\lambda_{0 ; m ; 0}$ (more precisely those with the largest real part governing the short time behavior) are not covered by UCNA; the other eigenvalues $\lambda_{i ; 0 ; n}$, however, governing the moderate-to-large time behavior of dynamical quantities, are well approximated by UCNA. In contrast to the case $\tau \rightarrow 0$, i.e. $\lambda_{l ; n=0 ; m=0}=l / \tau$ the asymptotic $\tau$-dependence, for $\gamma \tau \gg 1$ is correctly reproduced by UCNA.

The regions of validity of (5.4) and (5.5) are in agreement with the conditions $(3.6 \mathrm{a}, \mathrm{b})$ under which UCNA is derived. Fig. 1 shows a schematic plot of the region of validity of UCNA in the $\gamma-\tau$ parameter plane. This plot can be taken as a guide in checking the reliability of UCNA results of nonlinear systems for different parameter values.

Eigenvalues of the "small $\tau$ expansion". Applying the small- $\tau$ expansion to the FPE (2.5) with the parabolic potential $U(x)=1 / 2 \omega_{0}^{2} x^{2}$ one obtains in first order of $\tau$ a two-dimensional effective Fokker-Planck equation, see Appendix B and [13,29]. Besides the disadvantage of being incompatible with the standard small $\tau$ expansion of the overdamped dynamics $[16$, 22] as $\gamma \rightarrow \infty$, the eigenvalues do not depend on $\tau$ at all, i.e.

$\lambda_{n ; m}^{\mathrm{st}}=-\left(n \Lambda_{2}+m \Lambda_{3}\right)$

where $A_{2 ; 3}$ are given in (4.8c). Even in higher-orders of the small $\tau$ expansion (see Appendix B) the eigenvalues would remain uneffected by the noise correlation time $\tau$. As a consequence, dynamical quantities such as correlation functions do not exhibit a $\tau$-dependence. This is in clear contradiction to the exact solutions presented in Sect. 4.

\subsection{Stationary probability density and moments}

The stationary probability $P_{\mathrm{st}}^{\mathrm{UCNA}}(x, v)$ belonging to (5.1) is defined by

$P_{\mathrm{st}}^{\mathrm{UCNA}}(x, v)=Z^{-1} \exp \left(-\frac{\Phi^{\mathrm{UCNA}}(x, v)}{D}\right)$,

where $Z$ is a normalisation constant and $\Phi^{\mathrm{UCNA}}(x, v)$ is given by

$$
\begin{aligned}
& \Phi^{\mathrm{UCNA}}(x, v)=\frac{1}{2}\left(1+\gamma \tau+\tau^{2} \omega_{0}^{2}\right) \gamma v^{2} \\
& +\frac{1}{2}\left(1+\frac{\tau^{2} \omega_{0}^{2}}{1+\gamma \tau}\right) \gamma \omega_{0}^{2} x^{2}=\frac{1}{2}\left(\frac{D}{\left\langle x^{2}\right\rangle_{\mathrm{st}}} x^{2}+\frac{D}{\left\langle v^{2}\right\rangle_{\mathrm{st}}} v^{2}\right),
\end{aligned}
$$

which coincides with the exact two-dimensional probability $P_{\mathrm{st}}(x, v)=\int \mathrm{d} \varepsilon P_{\mathrm{st}}(x, v, \varepsilon)$ where $P_{\mathrm{st}}(x, v, \varepsilon)$ is given in $(2.6 \mathrm{a}, \mathrm{b})$. In contrast, using a small correlation time approximation scheme (see Appendix B) only the asymptotic small $\tau$ behavior is reproduced correctly.

\subsection{Correlation functions}

The normalized position-position correlation function $\left(\phi_{x x}(0)=1\right)$ of the approximated process (3.7) with the parabolic potential (4.1) is given by

$$
\begin{aligned}
& \phi_{x x}^{\mathrm{UCNA}}(t)= \\
& \left(\cos (\omega t)+\frac{\Gamma}{2 \omega} \sin (\omega t)\right) \exp \left(-\frac{\Gamma}{2} t\right) \\
& \left(\cosh (\omega t)+\frac{\Gamma}{2 \omega} \sinh (\omega t)\right) \exp \left(-\frac{\Gamma}{2} t\right) \\
& \left(1+\frac{\Gamma}{2} t\right) \exp \left(-\frac{\Gamma}{2} t\right) \quad \Gamma>2 \omega_{0} / \sqrt{1+\gamma \tau} \\
& \Gamma=2 \omega_{0} / \sqrt{1+\gamma \tau}
\end{aligned}
$$

where

$\omega=\left(\left|\omega_{0}^{2}-\frac{1}{4} \Gamma^{2}(1+\tau \gamma)\right| /(1+\gamma \tau)\right)^{1 / 2}$

and

$\Gamma=(\gamma /(1+\gamma \tau))\left[1+\left(\tau^{2} \omega_{0}^{2} /(1+\gamma \tau)\right)\right]$.

In Figs. 2-4 the exact position-position correlation functions are plotted (in normalized units [23]) against the approximations (5.10) for $\tau=0.1$ (Figs. 2), 


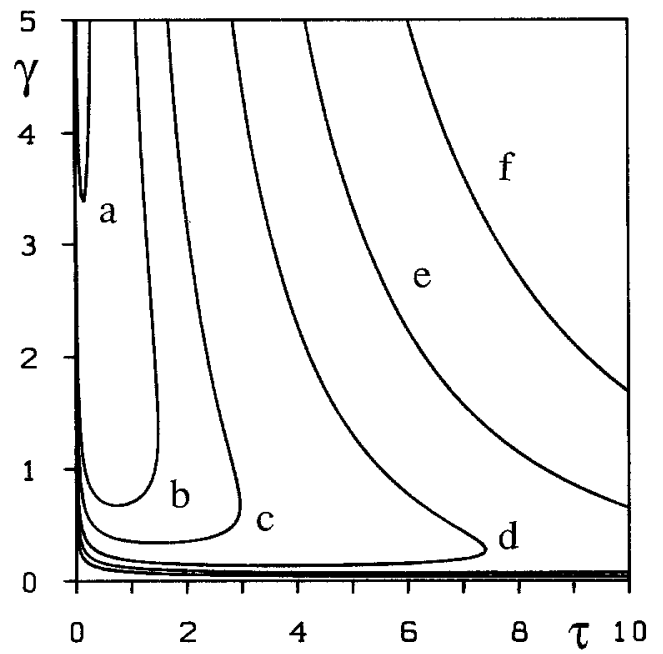

Fig. 1. Equation (3.6b) is shown as a contour-plot in the $\gamma-\tau$ parameter space for several values of the condition, i.e. we set for $D \tau \gamma^{2}(1+\gamma \tau)^{-3}$ the values (a) 0.5 , (b) 0.1 , (c) 0.04 , (d) 0.02 , (e) 0.01 , (f) 0.005 and draw the corresponding closed contourlines in the $\gamma-\tau$ parameter space. Note that for the smallest value $(f)$ in Fig. 1 the validity of the UCNA is obeyed best, because $\gamma$ and $\tau$ are large (upper right corner) and again along the $\tau$-axis for small $\gamma$ and along the $\gamma$-axis for small $\tau$

$\tau=10$ (Figs. 3) and $\tau=1$ (Fig. 4) for various values of the damping $\gamma$.

In the small $\tau$ regime (Fig. 2) we find excellent agreement for $\gamma=5$ and $\gamma=1$ and for $\gamma=0.1$. This behavior is well understood in view of the condition for the validity ( $3.6 \mathrm{~b}$ ) of the UCNA, see also Fig. 1.

For large correlation times $\tau$ (Figs. 3), the agreement is excellent for $\gamma=5$ and $\gamma=1$. For decreasing $\gamma$ the agreement remains excellent for the long-time tail. For intermediate times the oscillatory fine-structure of the exact correlation function is not reproduced by UCNA. As is best seen in Fig. $3 c(\gamma \tau=1)$, the UCNA still provides a coarse-graining of the fine structured exact correlation function.

For moderate $\tau$-values (Fig. 4) we also find excellent agreement for $\gamma=5$. For decreasing damping $\gamma$ the UCNA becomes invalid (see Fig. 1) and the agreement becomes worse. But even for $\gamma=1$ and $\tau=1$ the agreement is not very bad.

In conclusion the correlation functions are well approximated by UCNA in those parameter regions where the relevant eigenvalues are well approximated, or likewise, in parameter regions of the parameter space where the condition (3.6b) is obeyed (see Fig. 1).

\subsection{High friction limit}

For large values of the friction constant $\gamma$ the velocity $v$ can be eliminated adiabatically in $(2.4 \mathrm{a})$ (for precise
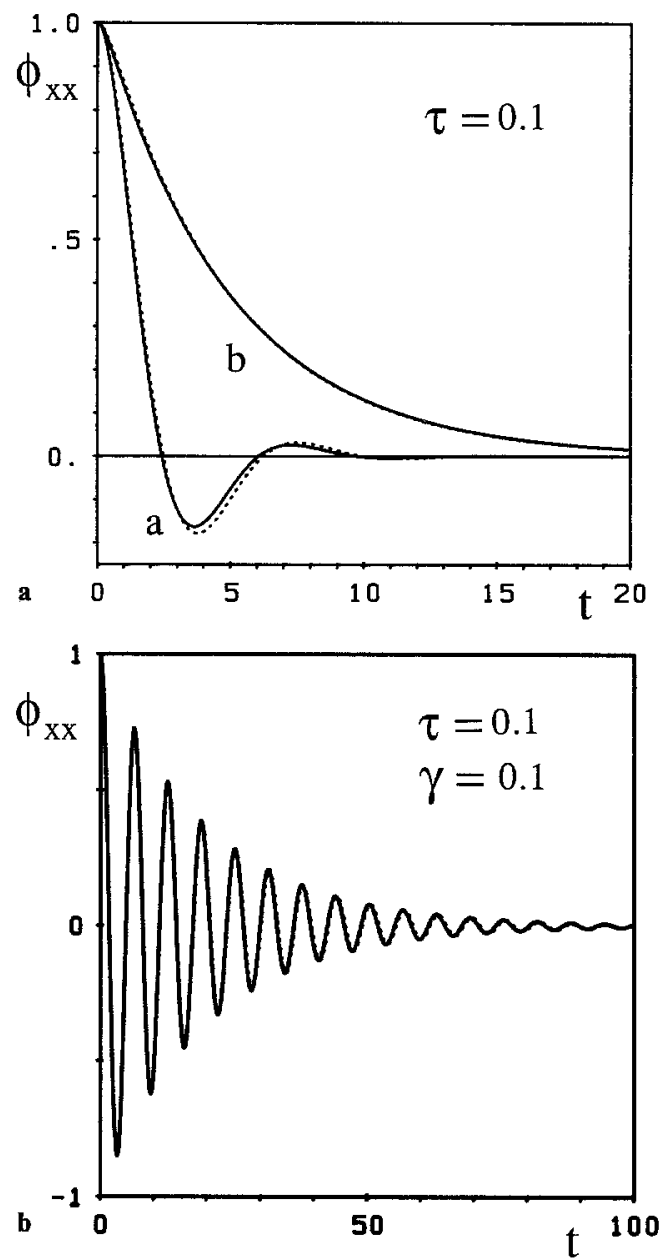

Fig. 2a and $\mathbf{b}$. The exact correlation functions (4.7) (solid) and the approximations (5.10) (dotted) are shown for $\tau=0.1$ at $\gamma=1$ (a) and $\gamma=5(\mathrm{~b})$ in $\mathbf{a}$ and at $\gamma=0.1$ in $\mathbf{b}$

conditions see [24]). Performing a time scale transformation $\tilde{t}=t / \gamma, \tilde{\tau}=\tau / \gamma$ we obtain the two-dimensional Markovian process

$$
\begin{aligned}
& \frac{\mathrm{d} x}{\mathrm{~d} \tilde{t}}=f(x)+\varepsilon \\
& \frac{\mathrm{d} \varepsilon}{\mathrm{d} \tilde{t}}=-\frac{1}{\tilde{\tau}} \varepsilon+\frac{\sqrt{\tilde{D}}}{\tilde{\tau}} \widetilde{\Gamma}(\tilde{t}) \\
& \left\langle\tilde{\Gamma}(\tilde{t}) \widetilde{\Gamma}\left(\tilde{t}^{\prime}\right)\right\rangle=2 \delta\left(\tilde{t}-\tilde{t}^{\prime}\right) .
\end{aligned}
$$

For the parabolic potential (4.1) the OU-process (5.11) can be solved exactly. The general case of a nonlinear force-field $f(x)$ has been discussed in [9] in terms of a numerical comparison, leading to the approximate Markovian process (3.8a). In the following we compare the analytical results for the eigenvalues, stationary correlation functions and relaxation times of the OU-process with the results of the UCNA (3.8a). The stationary distribution reproduced by UCNA is exact for the case of a parabolic potential. 

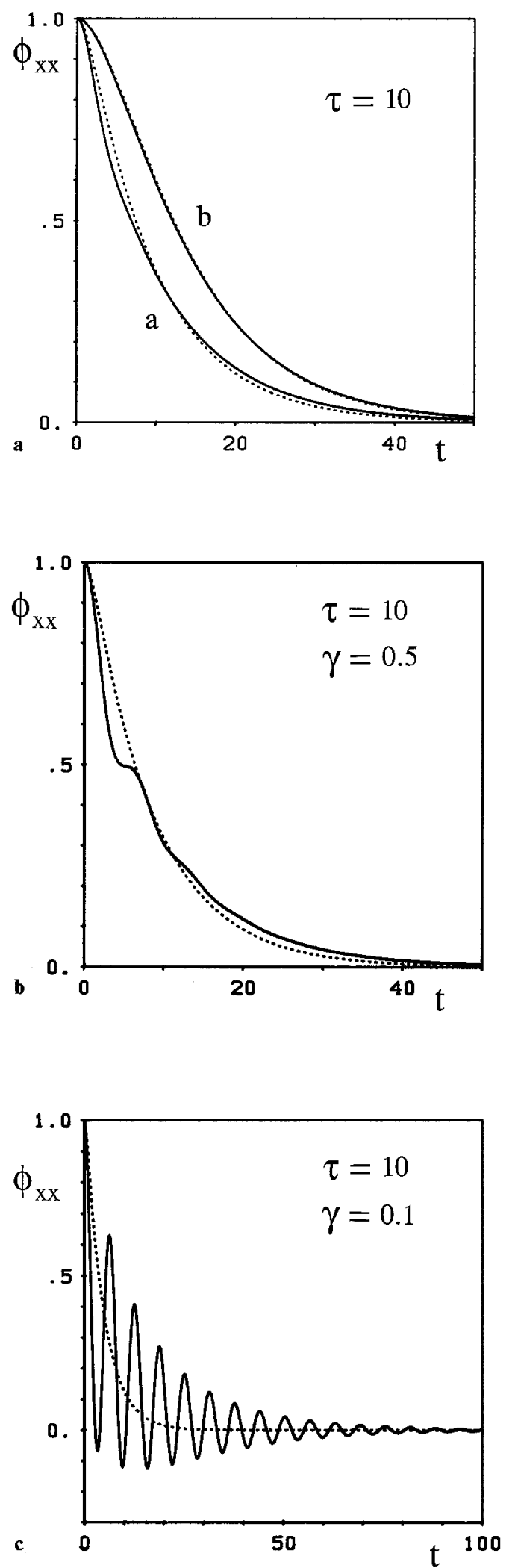

Fig. 3a-c. The exact correlation functions (4.7) (solid) and the UCNA approximations (5.10) (dotted) are shown for $\tau=10$ at $\gamma=1$ (a) and $\gamma=5$ (b) in a. In b (4.7) and (5.10) are presented for $\gamma=0.5$ and in $\mathbf{c}$ for $\gamma=0.1$

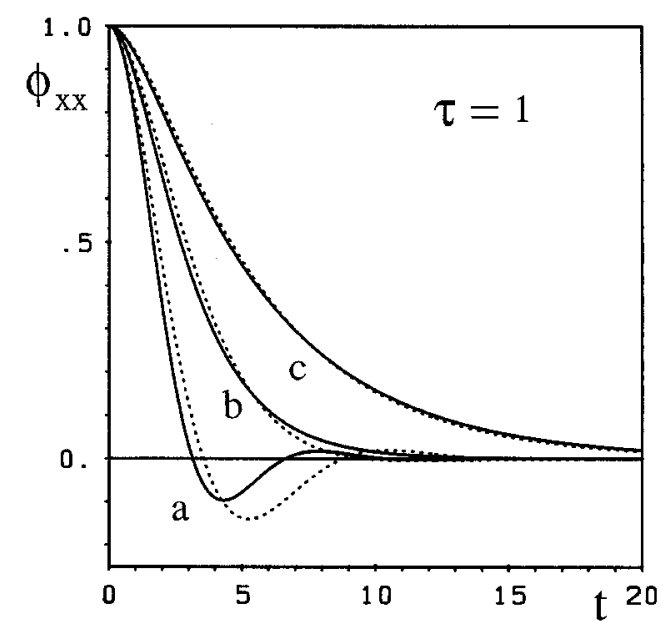

Fig. 4. The correlation functions (4.7) (solid) and (5.10) (dotted) are shown for $\tau=1$ and for $\gamma=1(\mathrm{a}), \gamma=2.5$ (b), $\gamma=5$ (c)
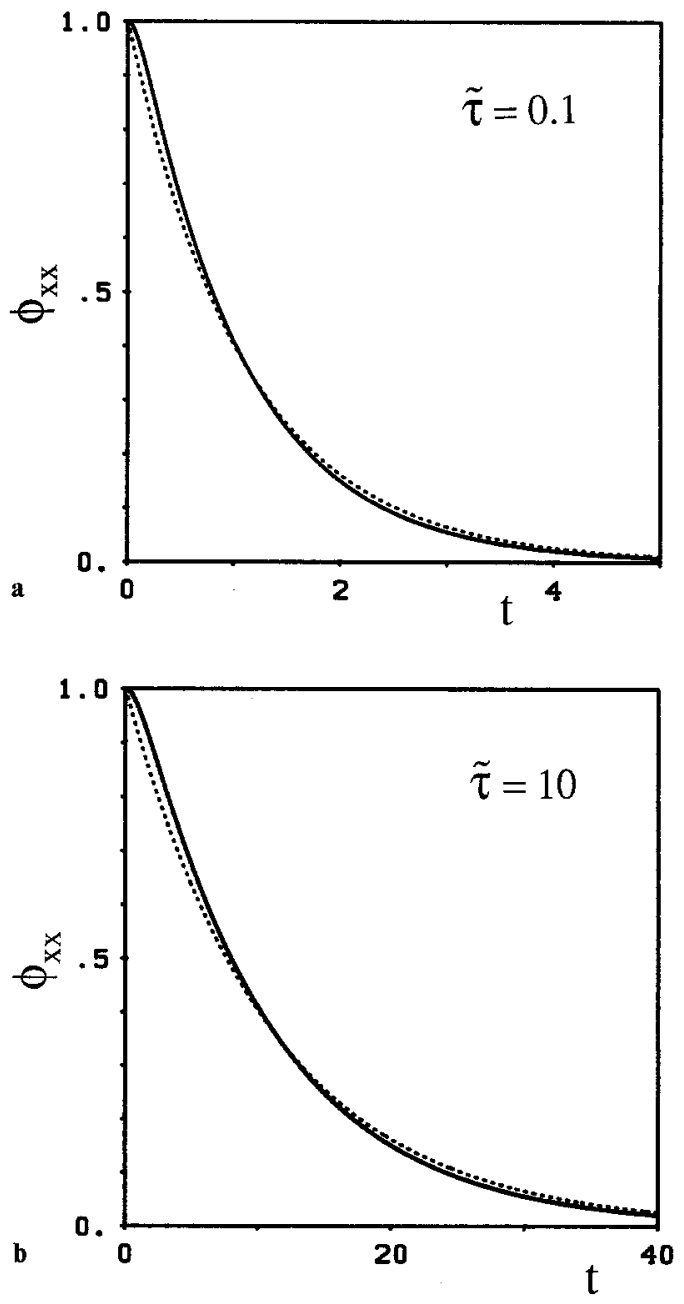

Fig. 5a and b. The exact correlation function in the overdamped case (5.14) (solid) and the UCNA approximation (5.15) (dotted) are shown for $\tilde{\tau}=0.1$ (a) and $\tilde{\tau}=10(\mathbf{b})$ in normalized units $\left(\omega_{0}=1\right)$ 
5.4.a. Eigenvalues. Using our technique in Appendix A the eigenvalues read

$\lambda_{n ; m}=n \frac{1}{\tilde{\tau}}+m \omega_{0}^{2}, \quad m, n=0,1,2, \ldots$

The one-dimensional UCNA approach provides

$\lambda_{l}^{\mathrm{UCNA}}=l \omega_{0}^{2} \frac{1}{1+\omega_{0}^{2} \tilde{\tau}^{2}}, \quad l=0,1,2, \ldots$

For $\tilde{\tau} \rightarrow 0 \lambda_{l}^{\text {UCNA }}$ approaches the smalles exact eigenvalues $\lambda_{n=0 ; m=l}$. Also in the limit $\tilde{\tau} \rightarrow \infty \lambda_{l}^{\mathrm{UCNA}}$ approaches the smallest exact eigenvalues which are in this case given by $\lambda_{n=l ; m=0}$. In both limits, $\tilde{\tau} \rightarrow 0$ and $\tilde{\tau} \rightarrow \infty$, UCNA picks out those eigenvalues which govern the moderate-to-long-time behavior.

5.4.b. Correlation functions. The normalized exact position-position correlation function of (5.11) equals the sum of two exponentially decaying contributions and is given by

$$
\begin{aligned}
\phi_{x x}(\tilde{t})= & \exp \left(-\omega_{0}^{2} \tilde{t}\right)+\tilde{\tau} \omega_{0}^{2} \frac{1}{\tilde{\tau} \omega_{0}^{2}-1} \\
& \cdot\left[\exp \left(-\frac{1}{\tilde{\tau}} \tilde{t}\right)-\exp \left(-\omega_{0}^{2} \tilde{t}\right)\right] .
\end{aligned}
$$

The UCNA provides instead the single exponential correlation function approximation

$\phi_{x x}^{\mathrm{UCNA}}(\tilde{t})=\exp \left(-\omega_{0}^{2} \frac{1}{\tilde{\tau} \omega_{0}^{2}+1} \tilde{t}\right)$.

The exact correlation function (5.14) has a vanishing initial slope $\dot{\phi}_{x x}(0)=0$ which is typical for a non-Markovian process (see for example [25]). This initial behavior can of course not be reproduced by our adiabatic (Markovian!) approach (5.15). Nevertheless the relaxation time defined as the integral over the normalized correlation function, i.e.

$T_{x x}=\int_{0}^{\infty} \phi_{x x}(\tilde{t}) \mathrm{d} \tilde{t}$

is reproduced exactly by UCNA

$T_{x x}^{\mathrm{UCNA}}=T_{x x}=\tilde{\tau}+\frac{1}{\omega_{0}^{2}}$.

In Figs. $5 \mathrm{a}, \mathrm{b}$ the exact correlation function (5.14) is plotted against the approximation (5.15) for $\tilde{\tau}=0.1$ and $\tilde{\tau}=10$. For $\tilde{\tau}=0.1$ (Fig. 5a) the agreement is excel- lent. For moderate $\tilde{\tau}$ the agreement becomes worse, whereas for large $\tilde{\tau}(\tilde{\tau}=10$, Fig. $5 b)$ the agreement is excellent again.

\section{Conclusions}

In this paper we have presented a novel approximation scheme (UCNA) for colored noise driven nonlinear systems with inertia. This scheme approximates the originally three-dimensional Fokker-Planck equation by a two-dimensional Markovian FPE. In contrast to the approximations in $[16,29]$ our novel UCNA (a Markovian approach) also reproduces reasonable well the dynamical quantities. We have applied the UCNA to the analytically solvable case of a parabolic potential. The stationary distribution coincides exactly, while eigenvalues and correlation functions agree excellently for a wide range of parameters, including small, moderate and large noise correlation times. For a nonlinear force-field, the error in the UCNA-approximation of stationary probabilities and moments originates thus solely from the nonlinear part of the force. Furthermore, the solution provides a good approximation for the local dynamics of all those nonlinear systems for which the potential $U(x)$ exhibits a parabolic curvature within one or possibly several regions of state space $(x, v)$ exhibiting an attracting fixed point.

As a byproduct we presented the exact eigenvalues and eigenfunctions of the $N$-dimensional OrnsteinUhlenbeck process (Appendix A). A point of further investigations is the application of our novel UCNA to a nonlinear force-field. A test of the UCNA, however, includes the numerical solution of the full threedimensional Fokker-Planck equation with natural boundary conditions, a task that is far from being trivial and which is beyond our present numerical capabilities. The numerical solution of the two-dimensional Fokker-Planck approach using our novel UCNA (3.7) may be obtained by the matrix continued-fraction technique [11].

\section{Appendix A: Eigenvalues and eigenfunctions of the $\mathrm{N}$-dimensional Ornstein-Uhlenbeck process}

To calculate the eigenvalues of a $\mathrm{N}$-dimensional OUprocess we start from the associated non-Hermitian Fokker-Planck operator $\underline{L}$

$$
L=\sum_{i ; j=1}^{N}\left(-\frac{\partial}{\partial x_{i}} M_{i j} x_{j}+D_{i j} \frac{\partial^{2}}{\partial x_{i} d x_{j}}\right)
$$


with the constant drift matrix $\underline{M}$ and the positive semi-definite diffusion matrix $\underline{D}$. The eigenvalues $\lambda$ and the right-hand eigenfunctions $\varphi_{\lambda}$ are defined by

$\underline{L} \varphi_{\lambda}=-\lambda \varphi_{\lambda}$.

The adjoint Fokker-Planck operator $\underline{L}^{\dagger}$

$\underline{L}^{\dagger}=\sum_{i ; j=1}^{N}\left(M_{i j} x_{j} \frac{\partial}{\partial x_{j}}+D_{i j} \frac{\partial^{2}}{\partial x_{i} \partial x_{j}}\right)$

has the same eigenvalues $\lambda$, but different eigenfunctions $\varphi_{\lambda}^{\dagger}$ (left-hand eigenfunctions), i.e.

$\underline{L}^{\dagger} \varphi_{\lambda}^{*}=-\lambda \varphi_{\lambda}^{\dagger}$.

Since the left hand eigenfunctions $\varphi_{\lambda}^{\dagger}$ are simple polynomials, (A.4) is more advantageous for the computation of the eigenvalues. For $\varphi_{\lambda}^{\dagger}$ the following Ansatz is used

$\varphi_{\lambda}^{\dagger}=R(\underline{x})+\sum_{m} c(\underline{m}) \prod_{i=1}^{N} x_{i}^{m_{i}}$,

where

$\underline{m}=\left(m_{1}, \ldots, m_{i}, \ldots, m_{N}\right), m_{i}=0,1, \ldots$

and the constraint

$\sum_{i=1}^{N} m_{i}=C>1$

The second term on the right-hand side of (A.5) is a sum over monomials of order $C$, the first is a polynomial of order $C-1$. The drift operator of $\underline{L}^{\dagger}$ maps a monomial of order $C$ onto another monomial of the same order. The diffusion operator maps the same monomial onto a monomial of order $C-2$. Since monomials are linear independent [26] we may disregard all monomials of lower order than $C$ and finally obtain the equation for the monomial of order $C$

$$
\sum_{i, j=1}^{N} M_{i j} x_{j} \frac{\partial}{\partial x_{j}} \sum_{m} c(\underline{m}) \prod_{i=1}^{N} x_{i}^{m_{i}}=-\lambda \sum_{\underline{m}} c(\underline{m}) \prod_{i=1}^{N} x_{i}^{m_{i}}
$$

where the constraint in (A.7) is obeyed.

If we assume that $\underline{\underline{M}}$ can be brought to a diagonal form by a similarity transformation $\underline{\underline{M}}_{\text {Diag }}=\underline{\underline{T M}} \underline{\underline{\underline{T}}} \underline{\underline{T}}^{-1}$ (this is possible if the eigenvalues $A_{n}$ of $\underline{\underline{M}}$ are not degenerate) (A.8) may be written as

$$
\sum_{i=1}^{N} A_{i} \sum_{\underline{m}} \mathrm{~d}(\underline{m}) m_{i} \prod_{k=1}^{N} y_{k}^{m_{k}}=-\lambda \sum_{\underline{m}} d(\underline{m}) \prod_{i=1}^{N} y_{i}^{m_{i}},
$$

where $d(\underline{m})$ are the transformed expansion coefficients, and the new variables $\underline{y}=\left(y_{1}, y_{2}, y_{3}, \ldots\right)$ are defined by

$\underline{y}=\underline{\underline{T}} \underline{x}$.

The eigenfunction $\varphi$ corresponding to the eigenvalue $\lambda$ contains only one monomial of order $C$, therefore only one set of coefficients $d(m=n)$ in (A.9) is correct; other eigenfunctions correspond to different sets $(\underline{m}=\underline{n}(\lambda))$.

Choosing respectively correct sets of coefficients $\mathrm{d}(\underline{m})$ (which are determined below) and comparing equal powers in (A.9) yields

$\lambda_{n_{1} n_{2} n_{3} \ldots n_{N}}=-\sum_{i=1}^{N} n_{i} \Lambda_{i}, \quad n_{i}=0,1,2,3, \ldots$

If some of the eigenvalues of $\underline{\underline{M}}$ are degenerate, $\underline{\underline{M}}$ can be brought to a Jordan form [27] by a similarity transformation, i.e. $\underline{M}_{\mathrm{Jordan}}=\underline{\underline{T}} \underline{\underline{T}} \underline{\underline{T}}^{-1}$. Performing the similarity transformation of (A.8) we are led to an equation for the variables $\underline{y}=\underline{\underline{T}} \underline{x}$ similar to (A.9), but with additional terms due to the non-diagonal contributions of the drift operator. These additional terms, however, do not contribute to the leading powers in $y_{i}$. Again we find the same eigenvalues given in (A.11), our main result of this appendix.

Besides the eigenvalues we can also determine the eigenfunctions of the $N$-dimensional OU-process. For a certain eigenvalue $\lambda$, the left-hand eigenfunction $\varphi_{\lambda}^{\dagger}$ is calculated by inserting a polynomial (A.5) into (A.4) and comparing equal powers. The order of the polynomial is given by the index of the eigenvalue $\lambda$ (compare (A.7) and (A.9)).

Since generalized detailed balance is valid for the $\mathrm{N}$-dimensional OU-process [28 a] the right-hand side eigenfunctions $\varphi_{\lambda}(x)$ and the left-hand eigenfunctions are (apart from a sign) related by

$P_{\mathrm{st}}(\underline{x}) \varphi_{\lambda}(\underline{\varepsilon x})=\varphi_{\lambda}^{\dagger}(\underline{x})$,

where $P_{\text {st }}(\underline{x})$ is the stationary probability density and $(\varepsilon x)$ are the time reversed coordinates. Thus the righthand side eigenfunctions can be calculated from the stationary probability density and the left-hand side eigenfunctions.

\section{Appendix B: Small correlation time expansion for colored noise driven systems with inertia}

Starting from the FPE (2.5)

$\frac{\partial P}{\partial t}=\left(\mathbf{A}+\varepsilon \mathbf{B}+\mathbf{L}_{\varepsilon}\right) P$ 
with

$\mathbf{A}=-\frac{\partial}{\partial x} v+\gamma \frac{\partial}{\partial v} v-f(x) \frac{\partial}{\partial v}$,

$\mathbf{B}=-\frac{\partial}{\partial v}$,

$\mathbf{L}_{\varepsilon}=\frac{1}{\tau} \frac{\partial}{\partial \varepsilon} \varepsilon+\frac{D}{\tau^{2}} \frac{\partial^{2}}{\partial \varepsilon^{2}}$,

we apply the technique of Appendix A.1 in [11c] (generalized to three-dimensional Fokker-Planck equations). As the result one finds for large times up to the order $\tau^{2}$ (extension to higher orders is straightforward) the two-dimensional Fokker-Planck approach for $P(x, t)((\mathrm{A} 1.32 \mathrm{a})$ of $[11 \mathrm{c}])$

$\frac{\partial P}{\partial t}=\mathbf{L} P$

where

$$
\begin{aligned}
\mathbf{L}= & \mathbf{A}+D \mathbf{B}^{2}+\tau D \mathbf{B}[\mathbf{A}, \mathbf{B}]+D \tau^{2} \mathbf{B}[\mathbf{A},[\mathbf{A}, \mathbf{B}]] \\
& +D^{2} \tau^{2}\left(\mathbf{B}[[\mathbf{B}, \mathbf{A}], \mathbf{B}] \mathbf{B}+\frac{1}{2} \mathbf{B}^{2}[[\mathbf{B}, \mathbf{A}], \mathbf{B}]\right)+O\left(\tau^{3}\right)
\end{aligned}
$$

and $[\mathbf{A}, \mathbf{B}]=\mathbf{A B}-\mathbf{B} \mathbf{A}$.

The commutators in (B.2) read

$$
\begin{aligned}
& {[\mathbf{A}, \mathbf{B}] }=-\frac{\partial}{\partial \mathrm{x}}+\gamma \frac{\partial}{\partial \mathrm{v}}, \\
& {[\mathbf{A},[\mathbf{A}, \mathbf{B}]]=\gamma \frac{\partial}{\partial \mathrm{x}}-\gamma^{2} \frac{\partial}{\partial \mathrm{v}}-\mathrm{f}^{\prime} \frac{\partial}{\partial \mathrm{v}}, } \\
& {[\mathbf{B},[\mathbf{A}, \mathbf{B}]]=0 . }
\end{aligned}
$$

The same result could also be obtained by extending the functional technique of $[8 \mathrm{~b}]$ to this higher dimensional model. In first order of $\tau$ this has already been done in [16], and recently in [29].

The operator $\mathbf{L}$ in (B.2) is explicitely given by

$$
\begin{aligned}
\mathbf{L}= & -\frac{\partial}{\partial x} v+\gamma \frac{\partial}{\partial v} v-f(x) \frac{\partial}{\partial v} \\
& +D\left(1-\gamma \tau+\tau^{2} f^{\prime}+\tau^{2} \gamma^{2}\right) \frac{\partial^{2}}{\partial v^{2}}+\tau D(1-\tau \gamma) \\
& \cdot \frac{\partial^{2}}{\partial x \partial v}+O\left(\tau^{3}\right) .
\end{aligned}
$$

In contrast du UCNA this FPE does not correspond to a Markovian process due to the possibly negative diffusion coefficients in (B.4), which do not follow from a corresponding Markovian stochastic differen- tial equation. Nevertheless the stationary probability density can be calculated in first order of $\tau$ [16], i.e.

$P_{\mathrm{st}}^{(1)}(x, v)=\frac{1}{Z} \exp \left\{-\frac{\gamma}{D} U(x)-\frac{\gamma}{2 D(1-\gamma t)} v^{2}\right\}$.

Note, that the truncation of the $\tau$ expansion is only valid for small $\tau$ and small $\gamma$. In first order of $\tau, P_{\text {st }}(x)$ $=\int \mathrm{d} v P_{\mathrm{st}}(x ; v)$ is not affected by the correlation time $\tau[16]$.

In second order of $\tau$ the stationary distribution of (B.4) does not factorize into position and velocity distributions for a general potential $U(x)$. For the parabolic potential $U(x)=1 / 2 \omega_{0}^{2} x^{2}$, however, the stationary solution is readily found and reads

$$
\begin{aligned}
& P_{\mathrm{st}}^{(2)}(x, v)=\frac{1}{Z} \exp \left\{-\frac{\omega_{0}^{2} \gamma}{2 D\left(1-\omega_{0}^{2} \tau^{2}\right)} x^{2}\right. \\
& \left.-\frac{\gamma}{2 D\left(1-\gamma \tau-\omega_{0}^{2} \tau^{2}+\gamma^{2} \tau^{2}\right)} v^{2}\right\} .
\end{aligned}
$$

In order $\tau^{2}$ the probability density in $x$ is affected by the correlation time $\tau$. The variance

$\sigma_{x}^{2}=\frac{D}{\omega_{0}^{2} \gamma}\left(1-\omega_{0}^{2} \tau^{2}\right)+O\left(\tau^{3}\right)$

exhibits the same behavior as (4.3a) for small $\tau$. For larger values of $\gamma$, however, $\tau$ is not the shortest time scale any more. As a consequence one has to take into account all higher order terms $\propto \tau^{n}$ in the Fokker-Planck approach in order to make the alternate series in the denominator of the second exponent of (B.6) convergent.

The eigenvalues of (B.4), determined by its driftmatrix (see Appendix A), are not affected by the $\tau$ corrections. From the exact eigenvalues (4.8) we find that this is correct only if $\gamma \tau \ll 1$. Then all terms proportional to $\exp (-t / \tau)$ will decay faster then all other exponentials and merely result in an initial slip. To use (B.4) as a starting point of the adiabatic elimination of the velocity $v$ appears to be rather doubtful because then $\gamma$ must become large, and $\gamma \tau \ll 1$ cannot be maintained for finite though small $\tau$.

\section{Appendix C: Condition for the validity of UCNA}

To start out let us consider first the situation for the Kramers problem, i.e. we consider the nonlinear Brownian motion of a particle of unit mass

$\dot{x}=v$

$\dot{v}=-\gamma v-K(x)+(\gamma \theta)^{1 / 2} \xi(t)$, 
where $\theta=k T$, and $\xi(t)$ is Gaussian white noise with mean zero and correlation $\langle\xi(t) \xi(s)\rangle=2 \delta(t-s)$. We shall now focus on the regime of large damping $\gamma$, yielding the Smoluchowski limit. For large $\gamma$, the velocity rapidly settles down to a limit value $v_{0}(x)$ which is slaved by the slow variable $x(t)$. Thus we adiabatically eliminate the velocity $v$ by setting $i=0$, i.e. $v_{0}(x)=-K(x) / \gamma$. The relaxation of the velocity variable occurs on the length scale $l_{0}$, given by the "brake-path"

$l_{0}=\frac{\theta^{1 / 2}}{\gamma}$.

For a consistent adiabatic elimination the force $K(x)$ should vary only little on the length scale $l_{0}$, since otherwise $v_{0}(x)$ is not attained on the relaxation time scale $\tau_{r}=1 / \gamma$. Working within appropriate dimensionless variables $\left(\tilde{x}, \tilde{v}, \tilde{t}, \tilde{\gamma}, \tilde{\theta}, \widetilde{I}_{0}\right)$, this condition reads

$\left|\tilde{K}^{\prime}\right| \tilde{l}_{0} \ll 1$.

In the original variables the above condition reads, with an appropriate length-scale

$\left|K^{\prime}\right| l_{0} \ll 1$ [dimension $\left.K\right]$.

Applying the above reasoning to $(3.2 \mathrm{a}, \mathrm{b}, \mathrm{c})$ we obtain with $z=q+f(x) /(1+\gamma \tau)$

$\dot{v}=z$

$\dot{z}=-\frac{1}{\tau}(1+\gamma \tau) z+\frac{1}{\tau} K(v, x)+\frac{\sqrt{D}}{\tau} \xi(t)$.

Hereby, we have neglected the change in the force field induced by $(\partial f / \partial x) \dot{x}$, since $x$ varies slowly compared to $v(t)$ and $q(t)$. Thus the term $f(x) v \gamma \tau /(1+\gamma \tau)$ in $(3.2 \mathrm{c})$, which stems from the time-derivative of (3.1) has to be neglected consistently. Hence the effective force-field $K(v, x)$ reads

$K(v, x)=-\gamma v+f(x)$.

Performing the time-scale transformation $\bar{t}=t / \sqrt{\tau}$ we obtain with $\dot{z}=\ddot{v}$

$\ddot{v}+\left(\tau^{-1 / 2}+\gamma \tau^{1 / 2}\right) \dot{v}-K(v, x)=\frac{D^{1 / 2}}{\tau^{1 / 4}} \xi(\bar{t})$

or

$\left.\ddot{v}+\bar{\gamma} \dot{v}-K(v, x)=(\bar{\theta} \bar{\gamma})^{1 / 2}\right) \xi(\bar{t})$

with $\bar{\gamma}=\left(\tau^{-1 / 2}+\gamma \tau^{1 / 2}\right)$ and $\bar{\theta}=D(1+\gamma \tau)^{-1}$. Therefore, we find from (C.2)

$l_{0}=D \tau^{1 / 2}(1+\gamma \tau)^{-3 / 2}$.
Combining (C.4) and (C.8) we achieve an expression for the region of validity of the UCNA in the $\gamma-\tau$ plane, i.e.

$\frac{\gamma(D \tau)^{1 / 2}}{(1+\gamma \tau)^{3 / 2}} \ll 1$

For a graphical representation of (C.9) see Fig. 1.

\section{References}

1. a. Nordholm, K.S.J., Zwanzig, R.: J. Stat. Phys. 13, 347 (1975); b. Garcia-Colin, L.S., Rio, J.L. del: J. Stat. Phys. 16, 235 (1977); c. Grabert, H., Talkner, P., Hänggi, P.: Z. Phys. B - Condensed Matter and Quanta 26, 389 (1977); d. Grabert, H., Talkner, P., Hänggi, P., Thomas, W.: Z. Phys. B - Condensed Matter and Quanta 29, 273 (1978); c. Grabert, H., Hänggi, P., Talkner, P.: J. Stat. Phys. 22, 537 (1980)

2. Haken, H.: Synergetics. In: Springer Series in Synergetics, Vol. 1. Berlin, Heidelberg, New York,: Springer 1983

3. a. Short, R., Mandel, L., Roy, R.: Phys. Rev. Lett. 49, 647 (1982); b. Dixit, S.N., Sahni, P.S.: Phys. Rev. Lett 50, 1273 (1983); c. Schenzle, A., Graham, R.: Phys. Lett. 98 A, 319 (1983); d. Lett, P., Short, R., Mandel, L.: Phys. Rev. Lett. 52, 341 (1984); e. Fox, R.F., James, G.E., Roy, R.: Phys. Rev. A30, 2482 (1984); f. Jung, P., Risken, H.: Phys. Lett. 103 A (1984); g. Lett, P., Mandel, L.: J. Opt. Soc. B2, 1615 (1985); h. Hernandez-Machado, A., San Miguel, M., Kalz, S.: Phys. Rev. A31 (1985); i. Zhu, S., Yu, A.W., Roy, R.: Phys. Rev. A 34, 4333 (1986); j. Fox, R.F., Roy, R.: Phys. Rev. A35, 1838 (1987); k. Lett, P., Gage, E.C., Chyba, T.H.: Phys. Rev. A35, 746 (1987); 1. Yu, A.W., Agrawal, G.P., Roy, R.: Opt. Lett. 12 (1987); m. Jung, P., Hänggi, P.: J. Opt. Soc. Am. B5, 979 (1988)

4. a. Vogel, K., Risken, H., Schleich, W., James, M., Moss, F, McClintock, P.V.E.: Phys. Rev. A 35, 463 (1986); b. Vogel, K., Leiber, Th., Risken, H., Hänggi, P., Schleich, W.: Phys. Rev. A35, 4882 (1987)

5. Schenzle, A., Brand, H.: Opt. Common 27, 485 (1978)

6. a. Stratonovich, R.L.: Topics in the theory of random noise. Vol. I, p. 98. New York: Gordon \& Breach 1963; b. Lax, M.: Rev. Mod. Phys. 38, 541 (1966); c. Van Kampen, N.G.: Phys. Rep. 24C, 171 (1976); d. Sancho, J.M., San Miguel, M., Katz, S.L., Gunton, J.D.: Phys. Rev. A26, 1589 (1982); e. Fox, R.F.: Phys. Lett. 94A, 281 (1983); f. Lindenberg, K., West, B.J.: Physica A 128, 25 (1984); g. Malchow, H., Schimansky-Geier, L.: Noise and diffusion in bistable nonequilibrium systems. In: Teubner Texte. Vol. 5, pp 83-87, Berlin: Teubner 1985; h. Leiber, Th., Risken, H.: Phys. Rev. A38, 3789 (1988) Improved small correlation time expansions: i. Fox, R.F.: Phys. Rev. A33, 467 (1986); j. Fox, R.F.: Phys. Rev. A37, 911 (1988)

7. a. Luciani, J.F., Verga, A.D.: Europhys. Lett. 4, 255 (1987); J. Stat. Phys. 50, 567 (1988); b. Tsironis, T.S., Grigolini, P.: Phys. Rev. Lett. 61, 7 (1988); c. Hänggi, P., Jung, P., Marchesoni, F.: J. Stat. Phys. 54, 1367 (1989)

8. a. Hänggi, P., Mroczkowski, T.J., Moss, F., McClintock, P.V.E.: Phys. Rev. A32, 695 (1985); b. For a more recent review see Hänggi, P.: In: Noise in nonlinear dynamical systems. Moss, F., McClintock, P.V.E. (eds.), Vol. I, Chap. 9, p. 307-p. 328. Cambridge: Cambridge University Press 1989

9. Jung, P., Hänggi, P.: Phys. Rev. A35, 4464 (1987)

10. a. Jung, P., Risken, H.: Z. Phys. B - Condensed Matter 61, 367 (1985); b. Leiber, Th., Marchesoni, F., Risken, H.: Phys. Rev. Lett. 59, 1381 (1987); c. Jung, P., Hänggi, P.: Phys. Rev. Lett. 
61, 11 (1988); d. Leiber, Th., Marchesoni, F., Risken, H.: Phys. Rev. A38, 983 (1988)

11. a. Risken, H., Vollmer, H.D.: Z. Phys. B - Condensed Matter and Quanta 33, 297 (1979); b. Vollmer, H.D., Risken, H.: Z. Phys. B - Condensed Matter and Quanta 34, 313 (1979); c. Risken, H.: The Fokker-Planck equation. In: Springer Series in Synergetics. Vol. 18. Berlin, Heidelberg, New York: Springer 1984

12. a. Gwinn, E.G., Westervelt, R.M.: Phys. Rev. Lett 54, 1613 (1985) b. Teitsworth, S.W., Westervelt, R.M.: ibid 56, 516 (1986)

13. Moss, F., Hänggi, P., Manella, R., McClintock, P.V.E.: Phys. Rev. A 33, 4459 (1986)

14. Schimansky-Geier, L.: Phys. Lett. A 126, 455 (1988); A 129, 481 (1988) (Erratum)

15. Ebeling, W., Schimansky-Geier, L.: In: Noise in nonlinear dynamical systems. Moss, F., McClintock, P.V.E. (eds.) Vol. I, Chap. 8, p. 279. Cambridge: University Press 1989

16. Fronzoni, L., Grigolini, P., Hänggi, P., Moss, F., Manella, R., McClintock, P.V.E.: Phys. Rev. A33, 3320 (1986)

17. Marchesoni, F., Menichella-Suetta, E., Pochini, M., Santucci, S.: Phys. Rev. A37, 3059 (1988)

18. Moss, F., Marchesoni, F.: Phys. Lett. A 131, 322 (1988)

19. Grabert, H., Hänggi, P., Talkner, P.: Z. Phys. B - Condensed Matter and Quanta 26, 389 (1977); J. Stat. Phys. 22, 537 (1980)

20. Marchesoni, F.: Phys. Lett. A 101, 11 (1984)

21. Uhlenbeck, G.E., Ornstein, L.S.: Phys. Rev. 36, 823 (1930); b. Wang, M.C., Uhlenbeck, G.E.: Rev. Mod. Phys. 17, 323 (1945)

22. Hänggi, P., Marchesoni, F., Grigolini, P.: Z. Phys. B - Condensed Matter 56, 333 (1984)

23. In all plots throughout this paper we scaled the state variables $x, v, \varepsilon$ and the parameters $\gamma, \tau$ dimensionless $(\bar{x}, \bar{v}, \bar{\varepsilon}, \bar{\gamma}, \bar{\tau}) \omega_{0}$ and, $D$ to be unity $\left(\bar{\omega}_{0}=1, \bar{D}=1\right)$. The scale transforms read $\bar{x}=\left(\omega_{0}^{3} / D\right)^{1 / 2} x, \bar{\varepsilon}=\left(D / \omega_{0}\right)^{1 / 2} \varepsilon, \bar{t}=\omega_{0} t, \bar{\gamma}=\gamma / \omega_{0}, \bar{\tau}=\omega_{0} \tau$

24. a. Becker, R.: Theorie der Wärme. Sect. 82, Berlin, Heidelberg, New York: Springer 1985; b. Chandrasekhar, S.: Rev. Mod. Phys. 15, 1 (1943); c. Kramers, H.A.: Physica 7, 284 (1940)
25. Jung, P., Risken, H.: In: Optical instabilities. In: Chambridge Studies in Modern Optics. Boyd, R.W., Raymer, M.G., Narducci, L.M. (eds.) Vol. 4, p. $361 \mathrm{ff}$. Cambridge: Cambridge University 1986

26. Lang, S.: Algebra, pp. $110 \mathrm{ff}$. Reading: Addison Wesley Publ. Comp. 1971

27. Wilkinson, J.H.: The algebraic eigenvalue problem. Oxford: Clarendon Press 1965

28. a. Hänggi, P., Thomas, H.: Phys. Rep. 88C, 207 (1982), see p. 272 ; b. Gardiner, C.W.: Handbook of stochastic methods. In: Springer Series in Synergetics, Vol. 13. Berlin, Heidelberg, New York: Springer 1985

29. Ramirez-Piscina, L., Sancho, J.M.: Phys. Rev. A37, 4469 (1988)

L. H'walisz, P. Jung, P. Hänggi

Institut für Mathematik

Universität Augsburg

Memminger Strasse 6

D-8900 Augsburg

Federal Republic of Germany

P. Talkner

Institut für Theoretische Physik

Universität Basel

Klingelbergstrasse 82

CH-4056 Basel

Switzerland

L. Schimansky-Geier

Sektion Physik

Humboldt-Universität zu Berlin

Invalidenstrasse 42

DDR-1040 Berlin

German Democratic Republic 